\title{
A Genetic Approach to Analyze Algorithm Performance Based on the Worst-Case Instances*
}

\author{
So-Yeong Jeon, Yong-Hyuk Kim
}

Department of Computer Science and Engineering, Kwangwoon University, Seoul, Korea.

Email: presentover@gmail.com, yhdfly@kw.ac.kr

Received June $29^{\text {th }} 2010$; revised July $15^{\text {th }} 2010$; accepted July $29^{\text {th }} 2010$.

\begin{abstract}
Search-based software engineering has mainly dealt with automated test data generation by metaheuristic search techniques. Similarly, we try to generate the test data (i.e., problem instances) which show the worst case of algorithms by such a technique. In this paper, in terms of non-functional testing, we re-define the worst case of some algorithms, respectively. By using genetic algorithms (GAs), we illustrate the strategies corresponding to each type of instances. We here adopt three problems for examples; the sorting problem, the 0/1 knapsack problem $(0 / 1 \mathrm{KP})$, and the travelling salesperson problem (TSP). In some algorithms solving these problems, we could find the worst-case instances successfully; the successfulness of the result is based on a statistical approach and comparison to the results by using the random testing. Our tried examples introduce informative guidelines to the use of genetic algorithms in generating the worst-case instance, which is defined in the aspect of algorithm performance.
\end{abstract}

Keywords: Search-Based Software Engineering, Automated Test Data Generation, Worst-Case Instance, Algorithm Performance, Genetic Algorithms

\section{Introduction}

In search-based software engineering, researchers have been interested in the automated test data generation so that it would be helpful for testing the software. Since, in general, test data generation is an undecidable problem, metaheuristic search techniques have been used to find the test data. McMinn's survey [1] summarizes previous studies. In the part of non-functional testing, these studies had a bias to generate the test data that show the best/worst-case execution time. But if we analyze an algorithm, not the entire program, there can be many measures other than the execution time, in terms of non-functional testing.

Finding test data (or problem instances) for an algorithm is as important as finding those of the entire program. The reason is that algorithms in a program can affect the entire performance of the program and we can exploit problem instances for the algorithms in analyzing them. In fact, Johnson and Kosoresow [2] tried to find the worst-case instance for online algorithms, for the lower bound proof. Also, Cotta and Moscato [3] tried to find the worst-case instance for shell sort to estimate the lower bound of the worst-case complexity.

*The present Research has been conducted by the Research Grant of Kwangwoon University in 2010.
Nevertheless, to the best of our knowledge, such trials are currently quite fewer than those in the field of software testing. Of course, fore-mentioned trials are good as initiative studies. But the trials did not introduce various strategies for constructing metaheuristics to generate the worst-case instance.

Differently from the fore-mentioned studies, in this paper, we introduce various strategies to construct genetic algorithms (GAs) [4] $]^{1}$ in generating the worstcase (problem) instance which is defined in the aspect of algorithm performance. For this, we try to find the worst-case instance of three example problems for some algorithms; the (internal) sorting problem, the 0/1 knapsack problem $(0 / 1 \mathrm{KP})$, and the travelling salesperson problem (TSP). These are well-known problems and each can show different strategy to construct a GA. Since we tried not to use the problem-specific knowledge in the construction, our suggested GAs can be extended to generate the instances of other similar problems. For the sorting problem, we take as test algorithm not only shell sort but also many well-known

\footnotetext{
${ }^{1}$ For the sorting problem and the $0 / 1$ knapsack problem, strictly speaking, we use memetic algorithms [5], which are GAs combined with local search. But we mainly focus on the GA rather than local search algorithm. Without classification, we just call our searching algorithm $\mathrm{GA}$, in this paper.
} 
sorting algorithms; quick sort, heap sort, merge sort, insertion sort, and advanced quick sort. For the 0/1KP and the TSP, we test the algorithm based on a greedy approach, comparing to the known-optimal algorithms which are based on the dynamic programming.

The remaining part of this paper is organized in the following. In Section 2, we introduce GAs and the three adopted problems with their popular algorithms. Also we define the worst-case instance for each problem, in terms of non-functional testing. In Section 3, we present our GAs for finding the worst-case instance. In Section 4, we explain our experiment plan and the results. We make conclusions in Section 5.

\section{Search Technique and Three Problems with Algorithms}

\subsection{Search Technique: Genetic Algorithms}

The genetic algorithm (GA) [4] is a non-deterministic algorithm. A non-deterministic algorithm makes guesses, which may or may not be the answer of the problem the algorithm wants to solve. So, it consists of two phases; the guess phase and the evaluation phase. In the guess phase, guesses are made. In the evaluation phase, those guesses are evaluated by how close they are to the right answer. For evaluating guesses, the objective function is defined. This function takes a guess and returns the numerical value which indicates how close the guess is to the right answer. In other words, a deterministic algorithm searches for an object which maximizing the given objective function.

How does GA make a guess? By the principle of evolution. GA manages multiple guesses or individuals. We call the set of individual population. A new individual can be constructed by two operations; crossover and $m u-$ tation. The crossover takes two individuals and returns one new individual. This new one is made by assembling each pattern of the two individuals. The mutation takes one individual and returns the individual which has slightly changed pattern from the taken individual. A pattern is found in the representation of the individual. Thus, the way an individual is represented is closely related to the way of making individuals. GA substitutes new individuals for some part of the population; this operation is called replacement.

How do we select individuals (in the population) for the crossover and the mutation as the input? Which individuals should we replace? By the qualities and the fitnesses [4] of the individuals. These are evaluated in the evaluation phase. The quality of an individual is the return value of the objective function. The fitness of an individual is evaluated using some part of the population or the entire population, as well as the individual to be evaluated. The fitnesses (not qualities) of the individuals are directly used to selection; fitness evaluation strategies are designed to increase the chances that some individuals with low qualities are selected. Note that using only qualities for selecting individuals can lose the diversity of the population and narrow the search range of GAs. Using the above operations, GA evolves the population until given stop condition is satisfied.

On the other hand, a local search algorithm can be combined with a GA. Given an individual, the local search tries to find out the individual with the best quality near the given individual. A GA combined with local search algorithms is called a memetic algorithm (MA) [5].

Since we want to generate the worst-case instance, each individual is a problem instance. Also, we should define the objective function so that this function returns the value indicating how close given individual is to the worst case. Thus the definition of the function depends on our definition of the worst case. Also, the way an instance is represented and strategies of crossover, mutation, replacement, fitness evaluation, and stop condition should be defined.

\subsection{Sorting Problem}

In the sorting problem, we are given an array of elements and their comparison operator. The correct solution of this problem is a sorted array in ascending (or descending) order. For the sorting problem, we assume that the comparison between elements takes so long time that it controls the total execution time. An instance of the problem is an array of elements to be sorted where the size of the array is fixed. Let the worst-case instance (array) be the instance that needs the most element-comparisons to sort. In this paper, we will test the following well-known sorting algorithms: quick sort, merge sort, heap sort, insertion sort, shell sort, and advanced quick sort.

Quick sort and merge sort [6] use the divide-andconquer strategy. In the quick sort, a pivot element [6] is typically taken as the first element in the array to be divided into two partitions; we use the same method in the tested our quick sort. Heap sort [6] mainly uses a data structure called heap [6]. Insertion sort [6] inserts each element of the array into the already-sorted part of the array one by one. Shell sort [7] uses insertion sort repeatedly using sequence of increments [8]. In tested shell sort, we use the sequence as the reverse order of $\left\{h_{n}\right\}$, where $n \geq 0$ and $h_{n+1}=3 \times h_{n}+1$ with $h_{0}=1$, where the sequence is bounded above the size of sorted array. To improve the quick sort, the advanced quick sort [6] takes as a pivot element the median element of three elements which are randomly chosen from the array to be divided into partitions.

\subsection{Zero/One Knapsack Problem}

Let item $_{i}$ be an ordered pair $\left(v_{i}, w_{i}\right)$ where $v_{i}$ is its value and $w_{i}$ is its weight. For a given set $S=\left\{\right.$ item $_{1}$, item $_{2}, \ldots$, 
item $\left._{n}\right\}$, we want to put items in $S$ into the knapsack where the maximum capacity $W$ is given as $c_{w} \times \sum w_{i}$ where $i=1$, $2, \ldots, n$. Here $c_{w} \in(0,1)$ is called the weight coefficient. The optimal solution of the problem is the subset $A$ of $S$ such that $\sum v_{i}\left(\right.$ item $\left._{i} \in A\right)$ is maximized and $\sum w_{i} \leq W$ (item $\left._{i} \in A\right)$, where $\sum v_{i}\left(\right.$ item $\left._{i} \in A\right)$ is called the objective value.

An algorithm based on the greedy approach [9] for this problem orders items in non-increasing order according to the 'value per unit weight' or profit density. Then, it tries to put the items in sequence satisfying that the total weight of the knapsack does not exceed $W$.

We will test the above algorithm in terms of the objective value. An instance of this problem is the set $S$ where the size of $S$ and $c_{w}$ is fixed. Let the worst-case instance be the instance maximizing $(O-P) / O$, where $P$ is the objective value obtained by the above algorithm and $O$ is the objective value obtained by an optimal algorithm based on dynamic programming [9].

\subsection{Travelling Salesperson Problem}

A weighted, directed graph $G(V, E)$ is given, where $V=$ $\{1,2, \ldots, n\}$ is a set of cities. An edge $e(i, j) \in E$ weighted by $C_{i j}$ represents that it costs $C_{i j}$ to go from city to city $_{j}$. An example is given in Figure 1. The optimal solution for the TSP is the tour, which is a Hamiltonian circuit of $G$, that takes the lowest (optimal) total cost if the tour exists. For our test, we assume that there always exists at least one tour although the tour is too expensive. The graph $G$ can be represented as an $N \times N$ adjacency matrix as in Figure 1.

In a greedy approach we consider, we start from city . To make a tour, we visit the adjacent city to which we

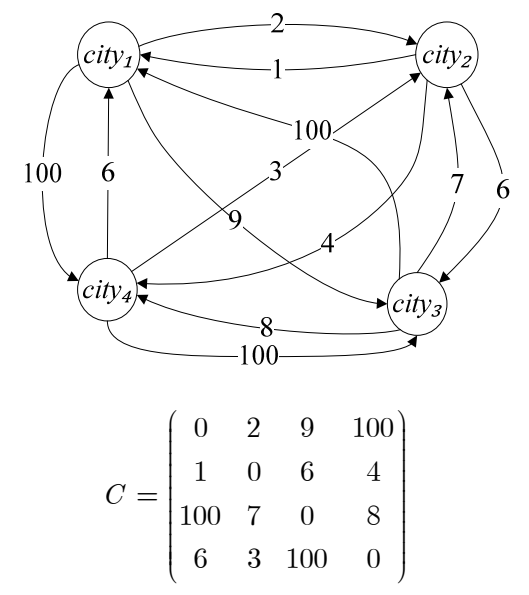

Figure 1. Two representations of an instance for TSP. NOTE. The cost to go from city to $_{1}$ city $y_{2}$ is 2 . This cost is the $[1,2]$-th element of the matrix $C$. On the other hand, the cost to go from city to $_{2}$ city $y_{1}$ is 1 . This cost is the [2, 1]-th element of the matrix $C$ can go from the current city at the lowest cost under the restraint that the city to go has not been visited before. Once the tour is constructed, 2-opt [10] improves this tour. Since the given graph is directed, two tours are derived from a base tour at one move in 2-opt; one derived tour is the reverse order of the other derived tour.

We will test an algorithm which uses the greedy approach with 2-opt in terms of the objective value (the tour cost). An instance of this problem is the graph $G(V$, $E)$ where the size of $V$ is fixed. So, let the worst-case instance be the instance maximizing $(P-O) / O$, where $P$ is the objective value obtained by the above algorithm and $O$ is the objective value obtained by an optimal algorithm based on dynamic programming [9].

\section{Genetic Algorithms}

\subsection{Framework and Things in Common}

There exist some trials to design somewhat different GAs [11-13] rather than just adopting traditional design. We also adopt a non-traditional framework of GAs. In our framework, the initialization of a population is done first. Then the following procedure is repeated until the stop condition we set is satisfied: 1) Fitness evaluation is done first. 2) Two parents are selected from the population. 3) One new individual is created by the crossover of the two parents. The other two new individuals are created by the local search from the mutation of each parent; one individual from one parent and another from the other parent. Steps 2) and 3) are repeated until sufficiently many new individuals are created. 4) Some individuals are replaced from the population with the new individuals.

The strategy of the population initialization, fitness evaluation, selection, replacement, and stop condition are fixed in our GAs. The strategies of other operations are different for each problem; these strategies will be introduced in the next sections. The initialization of the population is based on random generation. For the fitness evaluation, we used one based on population sharing [4]. The formula is as follows:

$$
F_{i}=f_{i} / \sum_{j \in\{1,2, \ldots, n\}} s\left(d_{i j}\right),
$$

where

- $F_{i}$ is the fitness of the $i$-th individual,

- $f_{i}$ is given as $\left(C_{i}-C_{\min }\right)+\left(C_{\max }-C_{\min }\right) /(k-1)$,

- $C_{i}$ is the quality of the $i$-th individual,

- $C_{\min }$ is the minimum quality among individuals,

- $C_{\max }$ is the maximum quality among individuals,

- $k(k>1)$ is a selective pressure,

- $n$ is the population size,

- $s\left(d_{i j}\right)$ is given as $1-\left(d_{i j} / \sigma\right)$,

- $d_{i j}$ is the distance between the $i$-th individual and the $j$-th one, and

- $\sigma$ is the longest distance among all $d_{i j}$ 's. 
Note that our definition of the distance is slightly different for each problem but is based on the Manhattan distance. By examining the formula, we can see that the fitness evaluation strategy helps to select the individuals far from other individuals of the population and thus keep the diversity of the population.

For the selection, we use fitness-proportionate selection using roulette wheel [4]. In the replacement, individuals with low qualities are replaced with new individuals regardless of the qualities of new ones. The stop condition is satisfied if the population reaches the given maximum number of generations, or all the individuals of the population become 'the same'. Strictly speaking, 'the same' means that the distance between every pair of individuals is zero. We take the individual with the highest quality among individuals in the final population. Then we say that the GA found the individual. In this paper, individuals are (problem) instances for a test algorithm.

\subsection{Sorting Problem}

We restrict that an instance of the sorting problem is a permutation of $N$ integers from 1 to $N$, where $N$ is fixed for every individual in a GA. A permutation is represented as an array. The sorting algorithms we deal with are to sort the given permutation in ascending order (i.e., $1,2,3, \ldots, N)$. For given sorting algorithm, the quality of an individual is the number of comparisons between elements needed to sort using the algorithm. Note that tested advanced quick sort uses pseudorandom-number generation and thus we repeat sorting the same permutation to take as the quality the average among the numbers of comparisons obtained by 50 repetitions. The distance between permutations $A$ and $B$ is defined as the sum of absolute differences between the numbers located in the same index of $A$ and $B$.

As the crossover of the permutation encoding, we use PMX $[14]^{2}$, which is popular. In our mutation of a permutation, say $A$, we decide a number at each index in $A$ to be swapped with another number at a randomly chosen index. Whether or not to swap is according to given probability, say $p_{m}$. For the local search from a permutation, we consider each pair of numbers in the permutation. We try to swap them and test that the new permutation has better a quality than the original one. If it is true, then the new one is substituted for the original one. Otherwise, we try to swap other pairs until the local search reaches the given maximum count of improvements.

\subsection{Zero/One Knapsack Problem}

An instance of the $0 / 1 \mathrm{KP}$ is a list of $N$ items. Here $N$ is fixed for every individual in the population. We represent

${ }^{2}$ Strictly speaking, we use the PMX introduced in the following URL: http://www.rubicite.com/Genetic/tutorial/crossover5.php. the list as an array. Note that an item is an ordered pair with value as its first coordinate and weight as its second coordinate; we represent an item as a two-dimensional point. We restrict the value and the weight are integers in $[1,100]$. The quality of an individual is $(O-P) / O$, where $P$ is the objective value obtained by the test algorithm and $O$ is the objective value obtained by an optimal algorithm; the more details are described in Subsection 2.3. To get the distance between two individuals (or lists), we first arrange items of each list in order of their profit densities. This is for normalization [15]. Then we obtain the distance as the sum of the absolute differences between the profit densities of items at the same position in the two lists.

In the crossover of two individuals (or lists), we also rearrange items of each list as in the calculation of distance. We derive each item in the offspring from the items at the same position in both parents. The illustration of deriving the item is shown in Figure 2(a). In the

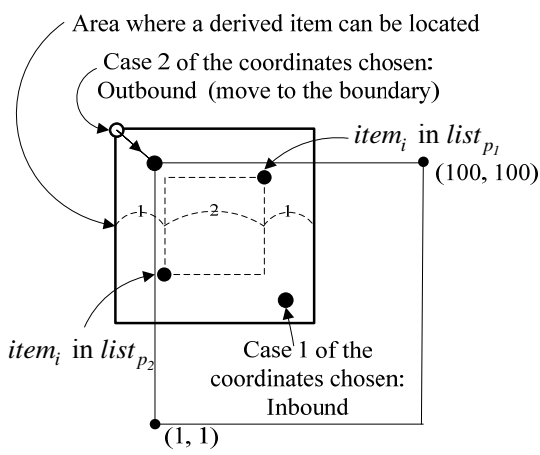

(a)

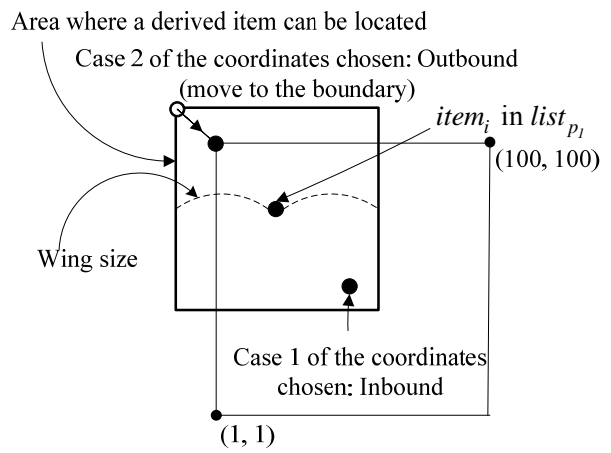

(b)

Figure 2. Crossover and Mutation in GAs for 0/1KP. (a) Crossover; (b) Mutation. NOTE. An item here is regarded as a two-dimensional coordinates. Part (a) is an illustration of deriving item $_{i}$ in offspring in crossover. Two item $_{i}$ 's in list $_{p_{1}}$ and list $_{p_{1}}$ make the area where item $_{\boldsymbol{i}}$ in offspring can be randomly chosen. Part (b) is an illustration of setting item $_{\boldsymbol{i}}$ in list $_{p_{1}}$ to be a new one in mutation. The square, whose center is item $_{\boldsymbol{i}}$ of list $_{p_{1}}$, indicates the area where item $_{\boldsymbol{i}}$ can be randomly chosen 
mutation from an individual, the probability of changing each item in the individual to be new one is $p_{m}$. Once an item is determined to be changed, we determine the area of the square where the point indicating a new item can be located. The center of the square is the point indicating the original item. The edge length of the square is determined using the following probability weight function of wing size or (edge length)/2: $y=a \times\left(x-x_{1}\right)+y_{1}$, where $a<0, y$ is the probability weight of choosing $x$ as a wing size, $y_{1}$ is the probability weight of choosing $x_{1}$, and $x_{1}$ is the maximum wing size. We set $y_{1}$ to be 1 and set $x_{1}$ to be floor $((U B-L B) / 2)$, with $U B=100$ and $L B=1$. For a given wing size, the illustration of the changing an item to be a new one is shown in Figure 2(b).

In the local search from an individual (or a list), we try to slightly move each item in the list and test that the new list has a better quality than old one. If it is true, then the new one is substituted for the original one. Otherwise, we try to move the next item in the list. The way of slightly moving the item $(v, w)$ is as follows: moving to $(v+1, w)$, $(v-1, w),(v, w+1),(v, w-1),(v+1, w+1),(v-1, w-1)$, $(v+1, w-1)$, or $(v-1, w+1)$, excluding one out of the boundary of $[1,100]$; we take the best way among all the possible ways.

\subsection{Travelling Salesperson Problem}

Every instance of the TSP is represented as an $N \times N$ adjacency matrix. The $N$, which is the number of cities, is fixed for every individual in the population. The $(i, j)$ element of the matrix is the cost to go from city co $_{i}$ tity. We restrict that every element of the matrix is an integer in $[1,100]$. The TSP does not limit the values to be integers. But the bounds reduce the time for finding the worst case, which is still helpful. We will try to find the worst-case instance for two different versions of the problem. In one version, the input instance $G$ is always represented as a symmetric matrix. In the other version, $G$ may be represented as an asymmetric matrix. The distance between two individuals is the sum of absolute differences between $(i, j)$ elements of two matrices. The quality of an individual is $(P-O) / O$, where $P$ and $O$ are described in Subsection 2.4.

For the crossover of two matrices, we use geographic crossover [16-18]. The geographic crossover can generate sufficiently diverse offspring with moderate difficulty in implementing. For the mutation from an individual (or a matrix), our approach is to move each $(i, j)$ element of the matrix with given probability $p_{m}$. We regard an element as a point on the number line. If an element is decided to be moved, the element can be moved randomly within the interval whose center is the original element. The interval size is decided by the probability weight function of the size. The function is quite similar to one in the $0 / 1 \mathrm{KP}$. The small size is taken more often than the big one. If we take large interval and the ele- ment moves out of $[1,100]$, we adjust the location to the closest boundary of the interval. We do not use the local search here.

\section{Experimental Results}

Using the framework and the strategies explained in Section 3, we tried to find the worst-case instances of test algorithms. We used the computer of which CPU model is Intel Core2 Duo T8100@2.10 GHz. In Table 1, we show the parameters in common for every GA. The usage of the mutation probability $\left(p_{m}\right)$ in Table $\mathbf{1}$ is described in Subsections 3.2, 3.3, and 3.4 respectively for the three test problems. Table 2 shows parameters in common for every random testing [1]. We ran the same GA fifty times to check whether our GAs are stochastically reliable. We say that two GAs are the same if and only if they are for the same problem and they belong to the same classification; we propose the classification in Tables 3-5, respectively for each problem. The weight coefficient in Table 4 is described in Subsection 2.3. The probability weight function of wing size in the same table is described in Subsection 3.3. The probability weight function in Table 5 is similar to one in Table 4. This function is referred to in Subsection 3.4.

Table 1. Parameters in common for every GA

\begin{tabular}{cc}
\hline Maximum number of generations & 1,000 \\
\hline Selective pressure & 3 \\
\# of individuals to be replaced for the next generation & 30 \\
Population size & 100 \\
Mutation probability $p_{m}$ & 0.15 \\
\# of independent runs of GA & 50 \\
\hline
\end{tabular}

Table 2. Parameters in common for every random testing

\begin{tabular}{cl}
\hline \# of instances randomly generated & 30,000 \\
\hline \# of independent runs of the random testing & 50 \\
\hline
\end{tabular}

Table 3. Classification and parameters of GAs for algorithms solving sorting problem

\begin{tabular}{|c|c|}
\hline \multirow{2}{*}{ Classification basis } & $\begin{array}{l}\text { Size of permutations: } \\
10,20,30 \text {, or } 40 \\
\text { (For advanced quick sort, we tested } \\
\text { only on size } 10 \text { and } 20 . \text {.) }\end{array}$ \\
\hline & $\begin{array}{l}\text { Sorting algorithm: } \\
\text { quick sort, merge sort, heap sort, in- } \\
\text { sertion sort, shell sort, or advanced } \\
\text { quick sort }\end{array}$ \\
\hline Elements of permutation & $\{1,2, \ldots, N\}$ \\
\hline $\begin{array}{l}\text { Limitation of improvement } \\
\text { counts in local search }\end{array}$ & $\begin{array}{l}\text { The smallest integer bigger than or } \\
\text { equal to } N \times 0.25+C_{n}^{k-1} \\
\text { ( } C_{n}^{k} \text { denotes the combination.) }\end{array}$ \\
\hline
\end{tabular}


Table 4. Classification and parameters of GAs for algorithms solving $\mathbf{0} / \mathbf{K P}$

\begin{tabular}{|c|c|}
\hline \multirow{2}{*}{ Classification basis } & $\begin{array}{l}\text { \# of given items: } 10,20 \text {, or } \\
30\end{array}$ \\
\hline & $\begin{array}{l}\text { Weight coefficient cw: } \\
0.25,0.5 \text {, or } 0.75\end{array}$ \\
\hline Range of value and weight of items & Integers in $[1,100]$ \\
\hline $\begin{array}{l}\text { Tangent slope of probability function } \\
\text { of wing size. }\end{array}$ & $\begin{array}{l}-1 \text { (wing size is an integer } \\
\text { from } 1 \text { to } 50 \text { ) }\end{array}$ \\
\hline
\end{tabular}

Table 5. Classification and parameters of GAs for algorithms solving TSP

\begin{tabular}{ll}
\hline \multirow{1}{c}{ Classification basis } & \# of cities: 5 or 10 \\
\cline { 2 - 2 } & $\begin{array}{l}\text { Kind of instances: symmetric } \\
\text { matrix or general matrix }\end{array}$ \\
\hline Range of weighted cost & Integers in $[1,100]$ \\
$\begin{array}{l}\text { Tangent slope of probability } \\
\text { function of interval size in } \\
\text { mutation. }\end{array}$ & -1 (interval size is an integer \\
f of cutting lines in a crossover 1 to 50$)$ & $0.5 \times$ (\# of cities) \\
\hline $\begin{array}{l}\text { NOTE. The crossover here is geographic crossover, which uses } \\
\text { cutting lines. }\end{array}$
\end{tabular}

After the run of a GA, we find one instance that shows the maximum quality in the final population; this instance is the closest to the worst case which we defined. Among those maximum qualities which are found by repeating the same GA fifty times, we get the average and the standard deviation. These values are in Figure 3, Figure 4, and Figure 5 respectively for each problem. Those figures are represented by the histogram with error bars; the length of error bar is 2 times the corresponding standard deviation. Some error bars in the figures are not identified because the corresponding standard deviation is close to 0. Note that in the figures, 'Avg' means the average, 'Std' means the standard deviation, 'Random' means the random testing method. In Figure 3, 'Ascend' means the permutation in ascending order (i.e., 1, 2, 3, $4, \ldots, N)$ and 'Descend' means the permutation in descending order (i.e., $N, N-1, \ldots, 4,3,2,1$ ). In Figure 5, 'symmetric TSP' means the TSP which does not allow any problem instance with an asymmetric matrix representation whereas 'general TSP' means the TSP which allows such problem instances. The CPU seconds taken by a run of the GA for each classification are given in Table 6, Table 7, and Table 8, respectively for each problem.

For test sorting algorithms, the quality of an individual (a permutation) was defined as the number of comparisons between elements when the permutation is sorted using the algorithm. In Figure 3, the worst cases of our quick sort, insertion sort, and advance quick sort takes more element comparisons than those of other test sorting algorithms. But seeing the result when the sorting
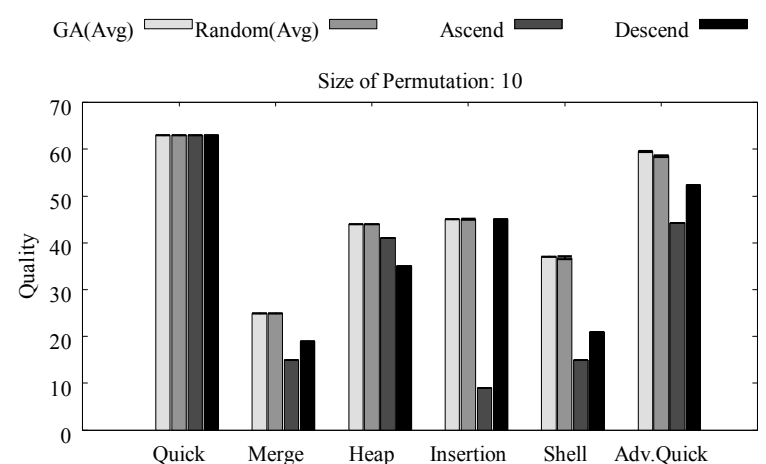

(a)

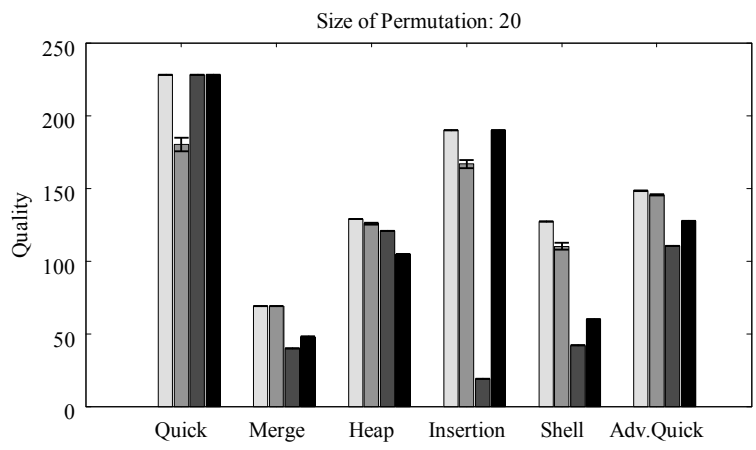

(b)

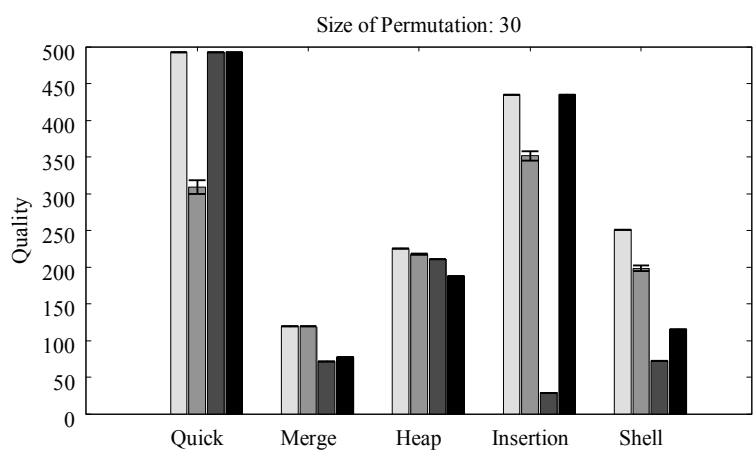

(c)

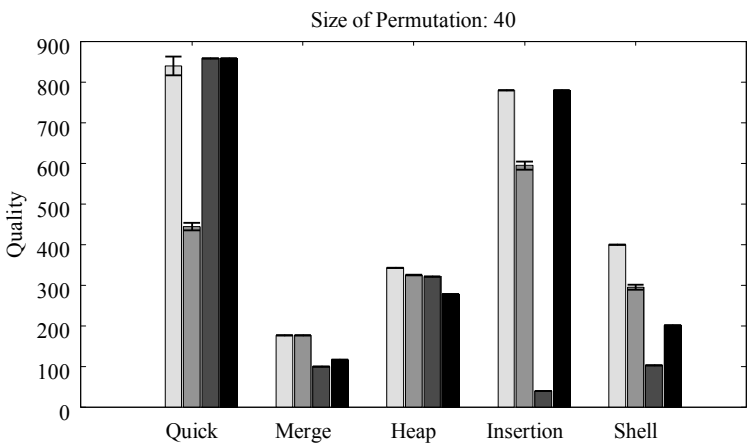

(d)

Figure 3. Quality of the worst-case instances (sorting problem). (a) Size of Permutation: 10; (b) Size of Permutation: 20; (c) Size of Permutation: 30; (d) Size of Permutation: 40. NOTE. The definition of the quality of an instance is given in Subsection 3.2 
GA(Avg) ${ }_{\text {Random(Avg) }} \longleftarrow$

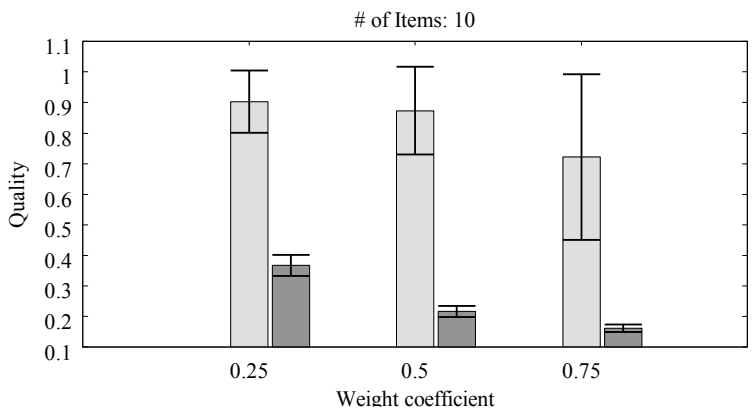

(a)

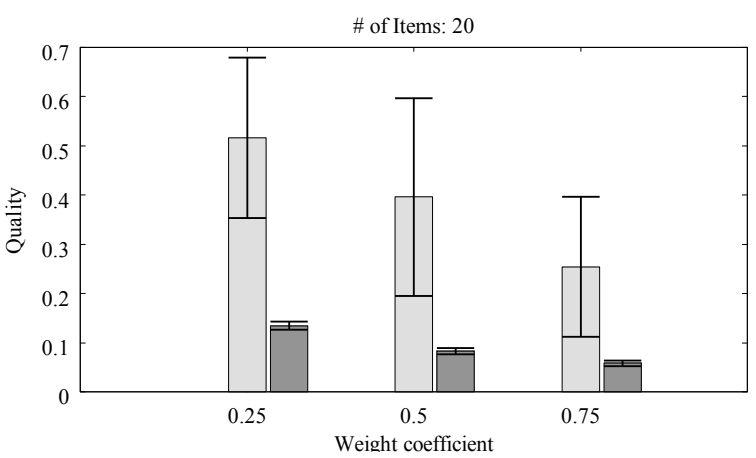

(b)

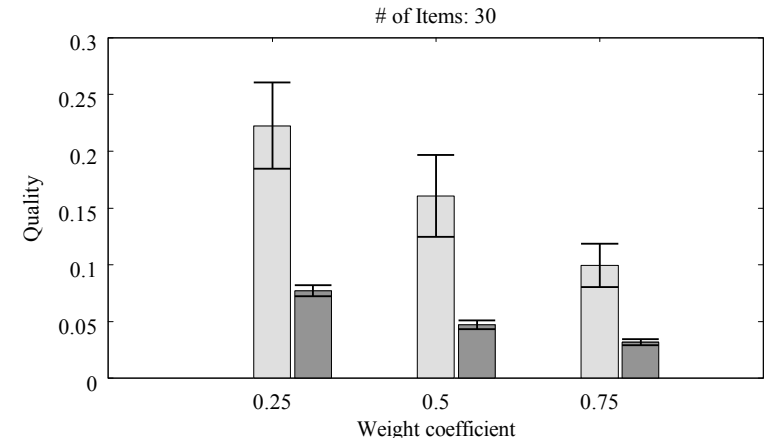

(c)

Figure 4. Quality of the worst-case instances (0/1KP). (a) The number of given items: 10; (b) The number of given items: 20; (c) The number of given items: 30 . NOTE. The definition of the quality of an instance is given in Subsection 3.3

Table 6. CPU seconds taken by a GA for each classification (sorting problem)

\begin{tabular}{ccccc}
\hline $\begin{array}{c}\text { Sorting algorithm / Size } \\
\text { of permutations }\end{array}$ & 10 & 20 & 30 & 40 \\
\hline Quick & 16 & 75 & 214 & 456 \\
Merge & 20 & 122 & 415 & 1,059 \\
Heap & 19 & 123 & 414 & 1,093 \\
Insertion & 19 & 134 & 496 & 1,359 \\
Shell & 17 & 85 & 282 & 729 \\
Advanced Quick & 1,352 & 12,502 & N/A & N/A \\
\hline
\end{tabular}

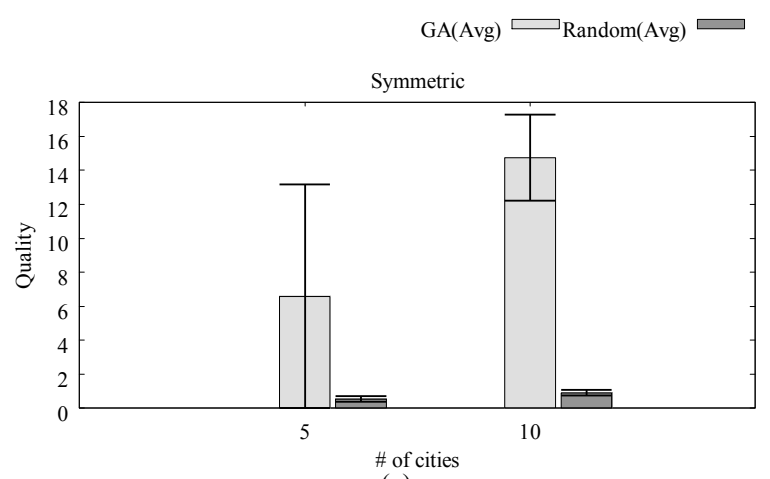

(a)

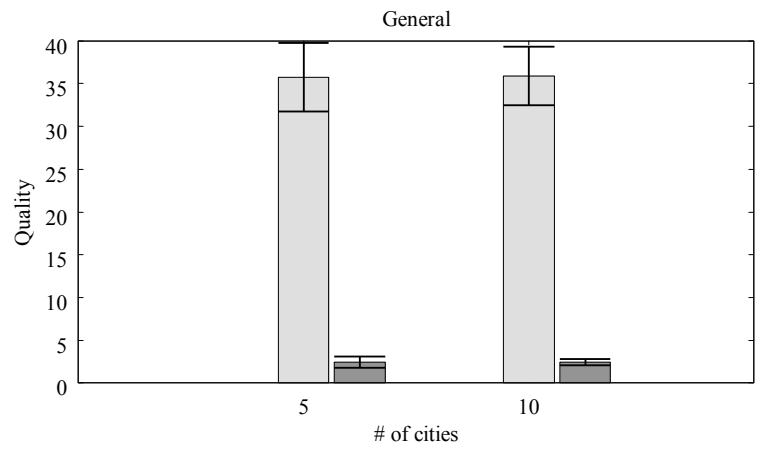

(b)

Figure 5. Quality of the worst-case instances (TSP). (a) The symmetric TSP; (b) The general TSP. NOTE. The definition of the quality of an instance is given in Subsection 3.4

Table 7. CPU seconds taken by a GA for each classification (0/1KP)

\begin{tabular}{cccc}
\hline $\begin{array}{c}\text { Weight coefficient / \# of } \\
\text { given items }\end{array}$ & 10 & 20 & 30 \\
\hline 0.25 & 70 & 607 & 2,415 \\
0.5 & 75 & 904 & 5,520 \\
0.75 & 105 & 1,927 & 6,000 \\
\hline
\end{tabular}

Table 8. CPU seconds taken by a GA for each classification (TSP)

\begin{tabular}{ccc}
\hline $\begin{array}{c}\text { \# of cities / } \\
\text { Kind of instances }\end{array}$ & $\begin{array}{c}\text { Symmetric } \\
\text { matrix }\end{array}$ & $\begin{array}{c}\text { General } \\
\text { matrix }\end{array}$ \\
\hline 5 & 18 & 18 \\
10 & 472 & 470 \\
\hline
\end{tabular}

permutation's size is 10 to 20 , we can predict that as the permutation size grows, the worst-case of our advanced quick sort takes fewer comparisons than that of our quick sort and insertion sort. On the other hand, the worst cases of merge sort, heap sort, and shell sort takes fewer comparisons than those of other algorithms.

For our test algorithm solving the 0/1KP (i.e., the algorithm based on a greedy approach which we explained in Subsection 2.3), the quality of an individual is $(O-P) / O$, where $P$ is the objective value obtained by the test algorithm and $O$ is the objective value obtained by an optimal algorithm. Regardless of the number of items 
(i.e., the magnitude of the searching space), the upper bound of the qualities is 1. We can see the result in Figure 4. For the same number of items, the higher the weight coefficient is, the lower the average quality of the worst cases found by our GA was. For the same coefficient, the more the given number of items is, the lower the average quality of the worst cases found by our GA was. When 10 items are given, the average quality of the worst cases was generally more than 0.7 ; our test algorithm is possibly not good enough in terms of optimality.

For our test algorithm solving the TSP (i.e., the algorithm based on a greedy approach with 2-opt which we explained in Subsection 2.4), the quality of an individual is $(P-O) / O$, the meaning of $O$ and $P$ is similar to those in the $0 / 1 \mathrm{KP}$. By examining the formula of the quality, we can assume that the upper bound of the qualities does not exist. In Figure 5, on the same number of cities, the average quality of the worst case in the symmetric TSP was lower than that of the worst case in the asymmetric TSP. The average quality of the worst case found by our GA in the symmetric TSP was about 14 when 10 cities are given, whereas one found in the asymmetric TSP was about 35 on the same condition; our test algorithm is generally not good enough in terms of optimality.

Overall, the results found by GAs were superior to those obtained by the random testing; we can conclude that our GAs have some effectiveness.

\section{Conclusions}

There are few trials to find the worst case for testing algorithms by GAs and the existing trials do not introduce various strategies for constructing GAs. Therefore, by taking as test examples the internal sorting problem, the $0 / 1 \mathrm{KP}$, and the TSP with some algorithms for each, we gave guidelines to the use of the GA in generating the worst-case instance for an algorithm. First, we defined the objective function of our GAs for the purpose of the analysis. In this paper, the objective function returns the quality of a problem instance; this quality indicates howclose the instance is to the worst case. Next, we introduced the framework of GAs and the specific strategies for each test problem.

For the sorting problem, we adopted as test algorithm quick sort, merge sort, heap sort, insertion sort, shell sort, and advanced quick sort. We defined the worst-case instance for a sorting algorithm as the instance that takes the most number of the element comparisons in using the algorithm. A problem instance can be represented as a permutation. We here used the PMX for the crossover. The mutation here swaps arbitrary elements in the permutation.

For the $0 / 1 \mathrm{KP}$ and the TSP, we tested the algorithm based on a greedy approach, comparing to an optimal algorithm. We defined the worst-case instance as the instance at which the test algorithm shows the most dif- ferent objective value from that obtained by the optimal algorithm. In the case of the $0 / 1 \mathrm{KP}$, a problem instance can be represented as the list of two-dimensional point whose coordinates are integers. Our suggested crossover is based on the idea of uniform crossover, but extended to two-dimensional version. The mutation runs point by point; each point in the list is moved to another close point with a high probability or to another far point with a low probability. In the case of the TSP, we represent a problem instance as a square matrix. We used the geographic crossover for the representation. The mutation runs element by element in the given matrix. Although the element here is just a scalar (not the two-dimensional point), the idea of mutation is similar to that of mutation for the $0 / 1 \mathrm{KP}$.

For the test algorithms, our GAs has some effectiveness as the experimental results are superior to those obtained by the random testing. The results of finding the worst cases show the following: 1) At the worst case, merge sort, heap sort, and shell sort takes fewer comparisons than other sorting algorithm. 2) Our greedy approach is not good enough in terms of optimality.

Our guidelines can help to analyze algorithms and can be used to test software. Since our guidelines are just for using GAs, we suggest giving guidelines for using other metaheuristics to generate the worst cases. Also note that many essential parts are still remained to analyze the algorithms. For future work, we suggest finding some weak points of a test algorithm and improve the algorithm by analyzing the worst-case instance.

\section{REFERENCES}

[1] P. McMinn, "Search-Based Software Test Data Generation: A Survey," Software Testing Verification And Reliability, Vol. 14, No. 2, 2004, pp. 105-156.

[2] M. Johnson and A. Kosoresow, "Finding Worst-Case Instances of, and Lower Bounds for, Online Algorithms Using Genetic Algorithms," Lecture Notes in Computer Science, Vol. 2557, 2002, pp. 344-355.

[3] C. Cotta and P. Moscato, "A Mixed-Evolutionary Statistical Analysis of an Algorithm's Complexity," Applied Mathematics Letters, Vol. 16, No. 1, 2003, pp. 41-47.

[4] D. Goldberg, "Genetic Algorithms in Search, Optimization, and Machine Learning," Kluwer Academic Publishers, Boston, 1989.

[5] P. Moscato, "Memetic Algorithms: A Short Introduction," In: D. Corne, M. Dorigo and F. Glover, Eds., New ideas in optimization, Mcgraw-Hill, London, 1999, pp. 219-234.

[6] M. Main and W. Savitch, "Data Structures and Other Objects Using C++," 3rd Edtion, Pearson/AddisonWesley, 2004.

[7] R. Sedgewick, "Algorithms in C, Parts 1-4: Fundamentals, Data Structures, Sorting, Searching," 3rd Edtion, Addison-Wesley, 1998. 
[8] D. Knuth, "The Art of Computer Programming, Volume 3: Sorting and Searching," 2nd Edtion, Addison-Wesley, New York, 1998.

[9] R. Neapolitan and K. Naimipour, "Foundations of Algorithms Using C++ Pseudocode," 3rd Edtion, Jones and Bartlett Publishers, Inc., 2008.

[10] C. Papadimitriou and K. Steiglitz, "Combinatorial Optimization: Algorithms and Complexity," Prentice-Hall, New Haven, 1981.

[11] L. Eshelman, "The CHC Adaptive Search Algorithm: How to Have Safe Search When Engaging in Nontraditional Genetic Recombination," In: G. Rawlins, Ed., Foundations of Genetic Algorithms, Morgan Kauffman, San Mateo, 1991, pp. 265-283.

[12] G. Harik, F. Lobo and D. Goldberg, "Compact Genetic Algorithm," IEEE Transactions on Evolutionary Computation, Vol. 3, No. 4, 1999, pp. 287-297.

[13] J. Grefenstette, "Genetic Algorithms for Changing Environments," Proceedings Parallel Problem Solving from Nature, Amsterdam, Vol. 2, 1992, pp. 137-144.
[14] D. Goldberg and R. Lingle, "Alleles, Loci, and the Traveling Salesperson Problem," Proceedings of the International Conference on Genetic Algorithms, Hillsdale, 1985, pp. 154-159.

[15] S. Choi and B. Moon, "Normalization in Genetic Algorithms," Genetic and Evolutionary ComputationGECCO-2003, Lecture Notes in Computer Science, Vol. 2723, Springer-Verlag, Berlin, 2003, pp. 862-873.

[16] T. Bui, B. Moon, "On Multi-Dimensional Encoding/ Crossover," Proceedings of the 6th International Conference on Genetic Algorithms, San Francisco, 1995, pp. 49-56.

[17] B. Kahng and B. Moon, "Toward More Powerful Recombinations," Proceedings of the 6th International Conference on Genetic Algorithms, San Francisco, 1995, pp. 96-103.

[18] C. Im, H. Jung and Y. Kim, "Hybrid Genetic Algorithm for Electromagnetic Topology Optimization," IEEE Transactions on Magnetics, Vol. 39, No. 1, 2003, pp. 2163-2169. 